\title{
Study of Rotating Axis Dynamic Characteristics
}

\author{
Ming-Hsiung $\mathrm{Ho}^{1, \star}$, Pin-Ning Wang ${ }^{2}$, Jung-Peng Yeh ${ }^{1}$, Ping-Hui Lee ${ }^{1}$ and Yan-Xiang Lai ${ }^{1}$ \\ ${ }^{1}$ Department of Mechanical Engineering, Taoyuan Innovation Institute of Technology, Jhongli, 32054, Taiwan \\ ${ }^{2}$ Department of Material and Fiber, Taoyuan Innovation Institute of Technology, Jhongli, 32054, Taiwan
}

\begin{abstract}
This study checks and analyses the design of a disk braking caliper bench testing machine, to testify its moving characteristics. The analysed objects include rotating axes and four weighting disks. The first procedure is to use the design software SolidWorks to build axis and weighting disks models. Next, the models are transferred to the finite element analysis software ANSYS to add boundary conditions and to conduct mode analysis. The ANSYS simulates axis and four weighting disks rotating stress analysis under 2000 RPM condition. The results show axis full-loaded rotating natural frequency is $74 \mathrm{~Hz}$ (4,440 RPM), the frequency is far away from working speed 2,000 RPM. The maximum stress is $67.9 \mathrm{MPa}$; when the stress is compared with material's ultimate stress is more than 10 times. The maximum displacement at disk is $0.056 \mathrm{~mm}$. The design should carefully check the tolerance between disk and machine cover. So the result of this design is safe. These design parameters can use for design check and machine manufacturing.
\end{abstract}

\section{Introduction}

With the progress of the computer aided engineering (CAE) software, the rapid growth of computer technology, the personal computer's calculation speed is faster than the past years. Finite Element Analysis (FEA) is a numerical technique that can be help to simulate the structural stress and mechanical vibrating systems. The Finite Element technique can also be assist for structural dynamic characteristics of studies of equipments. It has benefit to evaluate the dynamic characteristics of machines and structures prior to fabrication. FEA models could be used to approximate "structure stiffening" effects in rotating mechanical components such as fan wheels, pump rotors, motor shafts, etc. Therefore more finite element analysis; structural analysis in today design engineering, has become an inevitable step. FEA can help designers to modify the products shortcomings, and further to improve its design, with no error experiments by the repeated trial. Having this analysis information, it brings about to achieve cost savings and improves the structure design security.

Stetson [1] used of the first-order matrix transformation theory to predict the variation of natural frequencies and vibration damping system of non-shaped, while changing the amount of the solution best early design variables, in order to obtain the best analytical and experimental results correlation. Zhao [2] focused on the chuck shaft vibration problems in high speed operation conditions. The experimental modal analysis used to identify the modal frequency and vibration modes of the chuck shaft different cross-section in the constraint. The study found out that excitation point relationship to the modal frequency and the input excitation frequency. Sayer [3] analyzed the structure mechanical vibration characteristics by finite element numerical analysis method in order to understand what type damage failure caused by additional vibration source. The analysis method assists the design before the construction of the first assessment of the dynamic characteristics of the machine, FEA method can also simulate moving parts such as fans, rotors, rigid shaft stress stiffening under the influence of rotating machinery. Jana [4] analysed the nonlinear transverse vibrations of a rotating, clampedfree, flexible disc coupled to a pre-compressed spring. The disc was modelled as a Von Kármán plate, and the equations of motion are discretised by a Galerkin projection onto a pair of 1:1 internally resonant modes. The model could be representative of a large class of loadings in rotating disc systems such as air jet and electromagnetic excitation. Pusta etc. [5] concerned about the theoretical and numerical calculations of the flexural vibrations of a bladed disk. To elaborate the basic background for diagnostic and identification methods for ascertaining the main properties of the experimental model of turbine disks is the main project. By using damping heads to reduce of undesirable vibrations of blades is proposed, which on the experimental model of turbine disk are applied only on a limited number of blades.

In this study, the spindle dynamic analysis of vehicle braking performance test machine was conducted. Since the generated moment of inertia of the counterweight is attached on the machine axis when the spindle running. Various combinations of counterweight can provide a variety of braking performance and durability testings. Due to the configuration of the spindle with the counterweights, the stress and deformation effects on the operation of the spindle are taken into consideration. Therefore, through the above requirements, in this research will provide machine design recommendations, and check the machine's model characteristics before the formal design and manufacture.

\section{Methods}

Prior to FEA analysis, using SolidWorks CAD softwares building analysis models, then tranform models into IGS format for FEA analysis. The dynamic and rotational stress analysis wwere using ANSYS FEA software. After the final results of the post-process was demonstrated by the analysis software. 


\subsection{Analysis models and boundary conditions}

Computer simulation of structure dynamic properties is based on ANSYS finite element analysis software. Entity elements used in the model were solid type, spindle shape and the counterweights system based on the geometry of the design, created by SolidWorks software. The boundary conditions used refer to the designed spindle with the two bearings whch lie on two support positions. FEA analysis of the relevant basic information is as follows:

\subsubsection{Analysis model and material properties}

It is assumed that the spindle and counterweights model is homogeneous, isotropic, and continuity.

The main structure of this analysis as the main use of the four counterweights to meet the needs of different rotational moment of inertia. The models' material is set as SCM3. The material properties are listed in Table 1.

Table 1. The material properties for FEA.

\begin{tabular}{||c|c|c|c|c||}
\hline \hline DESIGNATION & \multicolumn{4}{|c||}{ PROPERTOES } \\
\hline \multirow{3}{*}{ GRADE } & $\begin{array}{c}\text { Young } \\
\text { Modulus } \\
\left(\mathrm{N} / \mathrm{mm}^{2}\right)\end{array}$ & $\begin{array}{c}\text { Yield } \\
\text { stress } \\
\mathrm{N} / \mathrm{mm}^{2} \\
\left(\mathrm{kgf} / \mathrm{mm}^{2}\right)\end{array}$ & $\begin{array}{c}\text { Tensile } \\
\text { Strength } \\
\mathrm{N} / \mathrm{mm}^{2} \\
\left(\mathrm{kgf} / \mathrm{mm}^{2}\right)\end{array}$ & $\begin{array}{c}\text { Poisson } \\
\text { Ratio }\end{array}$ \\
\hline HT & 210,000 & $\begin{array}{c}\geqq 686 \\
(70)\end{array}$ & $\begin{array}{c}\geqq 931 \\
(95)\end{array}$ & 0.3 \\
$($ HIGH TESILE) & & $(95)$ & \\
\hline \hline
\end{tabular}

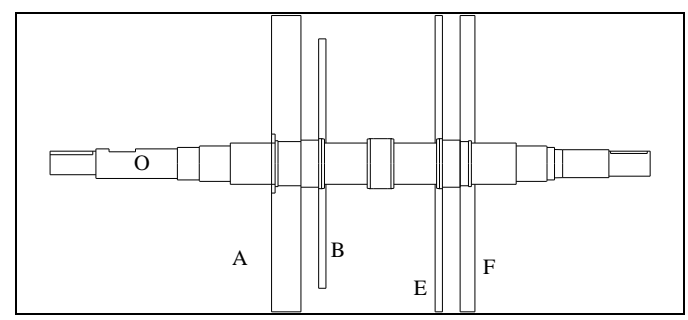

Figure 1. Rotation spindle and countweights

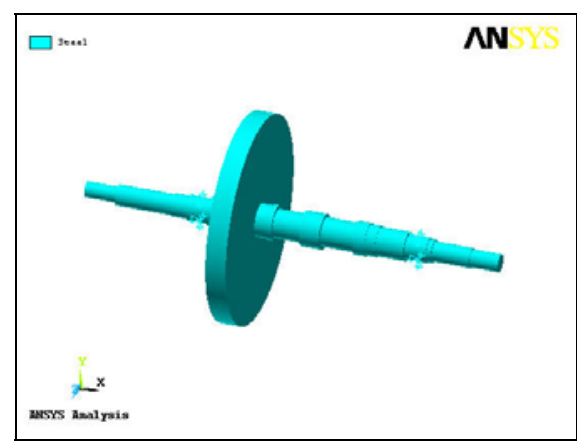

Figure 2. Boundary conditions.

\subsubsection{Boundary conditions}

This boundary conditions are set the model to close to the actual operation conditions. Fig. 1and Fig. 2 are showed the spindle and counterweights positions. The degree of freedom of bearing support position will be constrained to only make axial rotation, and can not be moved. This spindle maximum working speed is limited to 2,000 RPM.

For the analysis and discussion can be clearly identified, the spindle number is set as 0 , the counterweights sequentially numbered from left to right are set to A, B, E, F. The analysis arrangement will be 16 kinds of FEA models. The spindle and counterweights models are listed in Table 2.

Table 2. FEA models and mode analysis results.

\begin{tabular}{|c|c|c|c|}
\hline FEA models & \multicolumn{3}{|c|}{ Frequency (Hz) } \\
\hline No. & Mode 1 & Mode 2 & Mode 3 \\
\hline 0 & 336 & 547 & 564 \\
\hline $0-\mathrm{A}$ & 81 & 150 & 333 \\
\hline 0 -B & 139 & 160 & 185 \\
\hline 0 -E & 93 & 107 & 139 \\
\hline $0-\mathrm{F}$ & 114 & 147 & 208 \\
\hline $0-\mathrm{AB}$ & 79 & 140 & 164 \\
\hline $0-\mathrm{AE}$ & 81 & 105 & 119 \\
\hline $0-\mathrm{AF}$ & 81 & 123 & 133 \\
\hline $0-\mathrm{BE}$ & 93 & 108 & 131 \\
\hline $0-\mathrm{BF}$ & 111 & 141 & 159 \\
\hline $0-\mathrm{EF}$ & 95 & 110 & 141 \\
\hline $0-\mathrm{ABE}$ & 78 & 104 & 120 \\
\hline $0-\mathrm{ABF}$ & 77 & 123 & 156 \\
\hline $0-\mathrm{AEF}$ & 78 & 104 & 120 \\
\hline $0-\mathrm{BEF}$ & 93 & 97 & 110 \\
\hline $0-\mathrm{ABEF}$ & 74 & 103 & 120 \\
\hline
\end{tabular}

\subsection{Modal analysis}

The purpose of the modal analysis is to identify the model characteristics, and to expression the frequency with vibration shape. In frequency of resonance, the model will stimulate strong vibration distortion machine, and even make the machine component damage, and therefore the need to avoid or shift the resonance frequency. Fig. 2, the bearing support structure position to join binding condition design review provided by: Only axial rotation $(\mathrm{Rx})$, the rest of the direction of movement restrictions, this physical phenomenon is the use of bearing usage characteristics. Under this condition, the shaft and the counterweight combinations of models characteristics, (Modal Analysis) module is calculated by ANSYS modal analysis to identify the three resonant frequency and vibration shape.

\subsection{Rotation inertia stress analysis}

When the spindle and the counterweights under operation stage, the weights and inertia force of rotation cause the spindle and counterweights have stress and deformation. The binding conditions, such as using modal analysis, 
with added conditions the acceleration of gravity 9,800 $\mathrm{mm} / \mathrm{s}^{2}$ and machine maximum design speed is 2,000 RPM.

\section{Results and discussions}

\subsection{Modal analysis}

\subsubsection{Relationship between the resonance frequency and speed}

The results of modal analysis is to take the three mode frequencies. In this study, the relationship between rotation speed is frequency $(\mathrm{Hz})$ x $60=$ resonance speed (rpm), various combinations of counterweights resonant frequency are shown in Table 2.

\subsubsection{Frequency characteristics}

By the counterweights combination of resonance frequency Table 2, in which 0 -ABEF counterweight combination has lowest resonance frequency, because of the weight is the heaviest and difficult to operate the high-speed action, and its lowest resonance frequency is $74 \mathrm{~Hz}$, and converted to resonance excited rotational speed is $4,440 \mathrm{RPM}(74 \mathrm{~Hz} \times 60 \mathrm{~s})$, the speed is much greater than the operation speed of 2,000 RPM. Resonance phenomenon occurs in six degrees of freedom (Dx, Dy, Dz, Rx, Ry, Rz) in any direction, so the results of the analysis have different directions of vibration generated shape, Figure 3 is a 0 -ABEF counterweight combination $74 \mathrm{~Hz}$ with resonant shape.

Observation of the spindle model in the resonance frequency $336 \mathrm{~Hz}$, at about 20,000 RPM, the spindle is the basic element of each combination of counterweights.

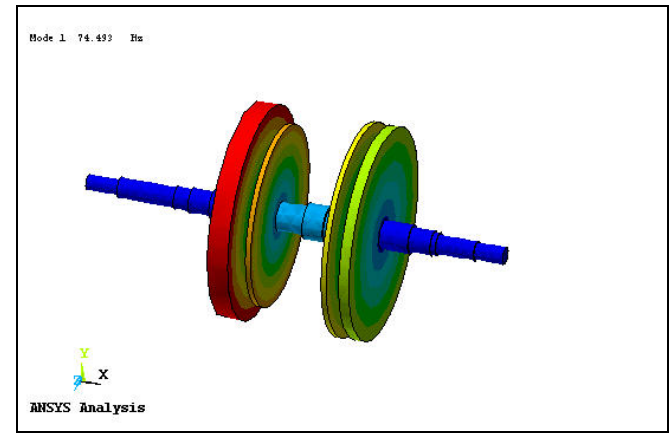

Figure 3. The mode shape of $0-\mathrm{ABEF}$ at $74 \mathrm{~Hz}$.

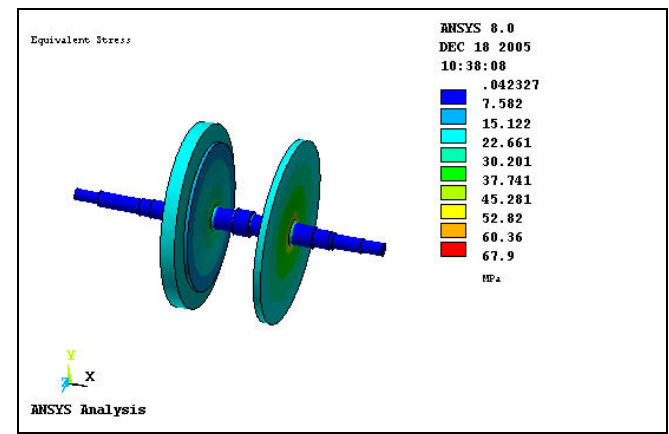

Figure 4. The 0-ABF stress at 2,000 RPM.

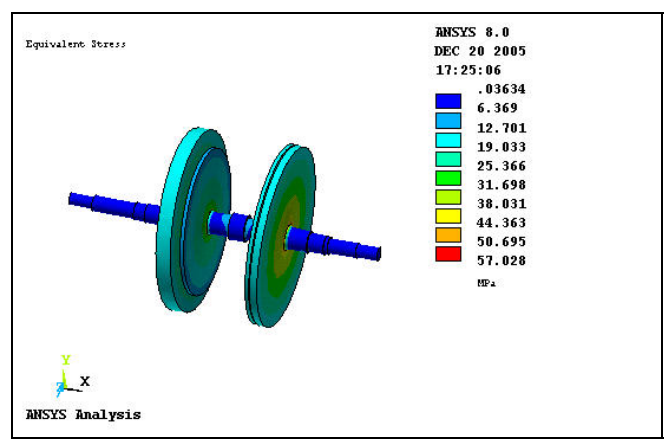

Figure 5. The 0-AEF stress at 2,000 RPM.

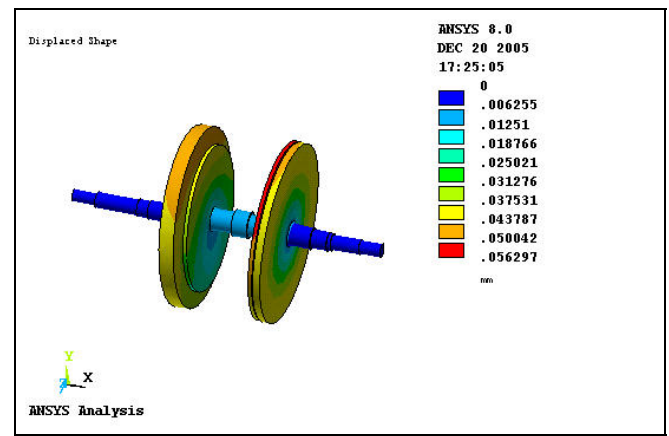

Figure 6. The deformation of $0-\mathrm{ABEF}$ at 2,000 RPM

\subsection{Stress analysis}

When the rotational speed of 2,000 RPM, the maximum stress and maximum deformation of various combinations of counterweights are shown in Table 4. Overall counterweights under 2,000 RPM, the spindle rotation stress is the minimum, but when coupled with weights, the stress concentration was generated significantly in the disk contacted with the shaft position. In Table 4, the $0-\mathrm{ABF}$ in the disk and shaft contact point had the maximum stress $67.9 \mathrm{MPa}$. The tensile strength was compared with the maximum stress, the safety factor was 10 or more, indicating that this design was safe in 2,000 RPM.

Where the largest concentration of stress Stress 0$\mathrm{ABF}$ and 0 -AEF, the rotational inertia caused by the most obvious, especially in the disk and spindle contact point. Both are characterized by close bearing support position, the fulcrum forces will focus, in particular weight deflection on both sides, so 0-ABF (Fig. 4) is similar to the 0-AEF (Fig. 5) counterweight combinations, the stress caused by values similar phenomenon.

General 0-ABEF had largest combination of cognitive inertia but does not produce the maximum stress, which was due to the weight distribution of the block at each position between the two bearings, stress concentration caused by the disk will be scattered in various the point of contact with the spindle, but the amount of displacement 0 -ABEF is still the biggest.

Analysis of the results of the stress values are lower than the tensile strength, the proposed shaft keyway groove or recess and the disk contact stress concentration at the position the design, in the design still need to pay attention to the strength and material selection. Counterweight disk edge displacement is worth noting, 
though, but to pay attention to each disk in the rotation with peripheral components need sufficient clearance.

Table 3. Analysis results of combinations of countweights.

\begin{tabular}{|c|l|c|c|}
\hline No. & $\begin{array}{c}\text { Counterweight } \\
\text { combination }\end{array}$ & $\begin{array}{c}\text { Maximum } \\
\text { stress } \\
\text { (MPa) }\end{array}$ & $\begin{array}{c}\text { Maximum } \\
\text { deformation } \\
(\mathrm{mm})\end{array}$ \\
\hline \hline 1 & 0 & 1.74 & 0.004 \\
\hline 2 & $0-\mathrm{A}$ & 60.60 & 0.046 \\
\hline 3 & $0-\mathrm{B}$ & 42.19 & 0.030 \\
\hline 4 & $0-\mathrm{E}$ & 56.45 & 0.049 \\
\hline 5 & $0-\mathrm{F}$ & 58.36 & 0.043 \\
\hline 6 & $0-\mathrm{AB}$ & 56.78 & 0.047 \\
\hline 7 & $0-\mathrm{AE}$ & 58.83 & 0.052 \\
\hline 8 & $0-\mathrm{AF}$ & 62.96 & 0.046 \\
\hline 9 & $0-\mathrm{BE}$ & 56.11 & 0.050 \\
\hline 10 & $0-\mathrm{BF}$ & 56.54 & 0.043 \\
\hline 11 & $0-\mathrm{EF}$ & 64.91 & 0.053 \\
\hline 12 & $0-\mathrm{ABE}$ & 57.05 & 0.052 \\
\hline 13 & $0-\mathrm{ABF}$ & 67.90 & 0.047 \\
\hline 14 & $0-\mathrm{AEF}$ & 67.28 & 0.055 \\
\hline 15 & $0-\mathrm{BEF}$ & 60.27 & 0.054 \\
\hline 16 & $0-\mathrm{ABEF}$ & 57.03 & 0.056 \\
\hline
\end{tabular}

\subsection{Deformation phenomenon}

Displacement results from the counterweight effect of the rotational inertia caused by the weight of the shaft and the counterweight when the disk rotates, due to the displacement caused by the disk axis yaw phenomenon, the end result may be the counterweight on the edge of change bit observed. In counterweight combination of 0 ABEF (Fig. 6), the counterweight block E had maximum deformation sharp. It had thin and large diameter disk, and easy to produce large displacement of instability.

\section{Conclusions}

1. The 0-ABEF counterweight combination had the lowest resonance frequency, the resonance frequencies into the critical speed 4,440 RPM $(74 \mathrm{~Hz}$ $\mathrm{x} 60 \mathrm{~s})$, the speed was much greater than the speed of 2,000 RPM.

2. Under the speed of 2000 RPM, It met the stress requirements, the maximum stress of $0-\mathrm{ABF}$ on the disk and spindle contact point was 67.90 MPa.

3. Stress analysis results showed that the stress of 0 $\mathrm{ABF}$ and 0 -AEF's largest rotational inertia caused by the stress concentration phenomenon is most obvious, especially in the disk and shaft contact point.

4. The reasons for displacement counterweight effect of the rotational inertia caused by the weight of the shaft, plus weights disk in the rotation, due to the displacement caused by the disk axis yaw phenomenon, the final result by the counterweight on the edge the displacement can be obtained. The 0 $\mathrm{ABEF}$ at the outer edge of the counterweight plate $\mathrm{E}$ had a maximum displacement position $0.056 \mathrm{~mm}$.

\section{References}

[1] K. A. Stetson and G. E. Palma, AIAA J. 14, 4 (1976)

[2] J. Zhao, J. C. Yuan, Adv. Mater. Res. 605-607 (2012)

[3] Sayer, Robert J., S. Vib. 38, 5 (2004)

[4] Anirban Jana, Arvind Raman, Non. Dyn. 40, 1 (2005)

[5] L. Pusta, L. Peseka, App. Comp. Mech. 5 (2011) 